\title{
Three-dimensional chemical analysis of laser-welded NiTi-stainless steel wires using a dual-beam FIB
}

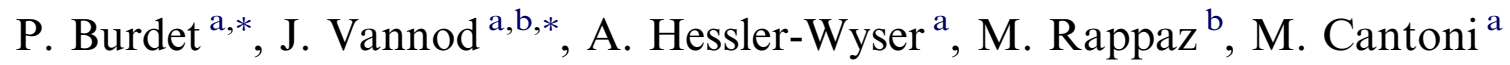 \\ ${ }^{a}$ Centre Interdisciplinaire de Microscopie Electronique, Ecole Polytechnique Fédérale de Lausanne (EPFL), CH-1015 Lausanne, Switzerland \\ ${ }^{\mathrm{b}}$ Laboratoire de Simulation des Matériaux, Ecole Polytechnique Fédérale de Lausanne (EPFL), CH-1015 Lausanne, Switzerland
}

Received 27 August 2012; received in revised form 31 January 2013; accepted 31 January 2013

Available online 26 February 2013

\begin{abstract}
The biomedical industry has an increasing demand for processes to join dissimilar metals, such as laser welding of NiTi and stainless steel wires. A region of the weld close to the NiTi interface, which previously was shown to be prone to cracking, was further analyzed by energy dispersive spectrometry (EDS) extended in the third dimension using a focused ion beam. As the spatial resolution of EDS analysis is not precise enough to resolve the finest parts of the microstructure, a new segmentation method that uses in addition secondaryelectron images of higher spatial resolution was developed. Applying these tools, it is shown that this region of the weld close to the NiTi interface does not comprise a homogeneous intermetallic layer, but is rather constituted by a succession of different intermetallics, the composition of which can be directly correlated with the solidification path in the ternary Fe-Ni-Ti Gibbs simplex.
\end{abstract}

(C) 2013 Acta Materialia Inc. Published by Elsevier Ltd. All rights reserved.

Keywords: Intermetallic phases; Laser welding; Focused ion beam (FIB); Energy dispersive X-ray spectrometry (EDS); 3-D image analysis

\section{Introduction}

Thanks to its properties (pseudoelasticity, shape memory, corrosion resistance and biocompatibility), nickel-titanium (NiTi) plays an important role in biomedical engineering. It is used, for instance, to design medical devices for invasive surgery such as catheter guide-wires, stents or coil anchors. In order to extend the range of applications and to reduce final product costs, there is a strong interest in joining NiTi with other biocompatible alloys such as stainless steel (SS).

In a previous study, the mechanical integrity of laserwelded joints between NiTi and SS wires was investigated [1]. In situ tensile tests in a scanning electron microscope revealed an unexpected fracture location: nucleating at

\footnotetext{
* Corresponding authors at: Centre Interdisciplinaire de Microscopie Electronique, Ecole Polytechnique Fédérale de Lausanne (EPFL), CH1015 Lausanne, Switzerland (J. Vannod). Tel.: +41 21 6934437; fax: +41 216934401 (P. Burdet).

E-mail address: pierre.burdet@a3.epfl.ch (P. Burdet).
}

the periphery of the welded region close to the NiTi interface, the crack first propagates with a brittle mode all around the wire at the onset of the superleastic plateau. At the end of this plateau, ductile rupture of the specimen occurs by propagation of the crack into the NiTi wire. It was shown that this behavior results from a stress concentration in the weld near the interface perimeter induced by three concomitant mechanisms: (i) the shape of the weld, which is wider at the surface and narrower in the middle of the wire; (ii) the higher elastic modulus of the weld compared to that of NiTi; and (iii) the radial contraction of the NiTi wire at the onset of the superelastic plateau (associated with the isovolumetric martensitic transformation). In order to assess that this early failure is not favored also by the formation of brittle intermetallics, an extended knowledge of the different phases and their location in the welded region close to the NiTi base wire is needed at the microscale.

A first step to apprehend the complex material microstructure that forms during heterogeneous laser welding consists in considering the multicomponent phase diagram. 


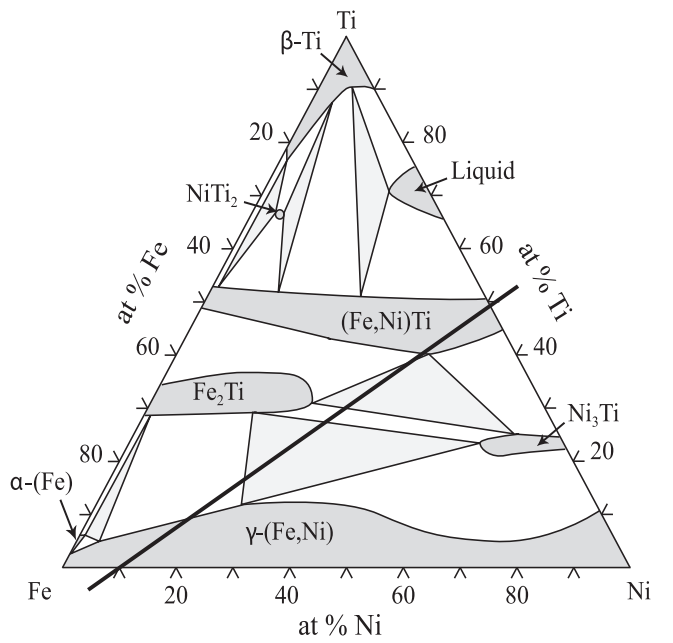

Fig. 1. Fe-Ni-Ti ternary phase diagram, cut isothermally at $1000{ }^{\circ} \mathrm{C}[2]$.

As $\mathrm{Cr}$ has a small influence on the welded joint composition as well as on the phase formation, ${ }^{1}$ the three main elements considered in this work are $\mathrm{Ti}, \mathrm{Fe}$ and $\mathrm{Ni}$. An isothermal cut at $1000^{\circ} \mathrm{C}$ of a ternary $\mathrm{Fe}-\mathrm{Ni}$-Ti phase diagram is shown in Fig. 1 [2]. The black line connects the two initial phases, the SS on the left corner and the NiTi on the middle right of the Gibbs simplex.

Following the diffusion path theory described by Kirkaldy et al. [3], the phases that can be present in the welded joint are those present in the single- or multi-phase domains crossed by the line linking the two initial materials. In the present case, the potential phases are $(\mathrm{Fe}, \mathrm{Ni}) \mathrm{Ti}, \mathrm{Ni}_{3} \mathrm{Ti}$, $\mathrm{Fe}_{2} \mathrm{Ti}$ and $\gamma-(\mathrm{Fe}, \mathrm{Ni})$. During the sample production, the base wires are melted to form the welded area. A mixing process mainly driven by Marangoni convection takes place, leading to a complex microstructure, both in a geometrical and compositional sense. With such a complex system, an accurate characterization technique that is able to chemically identify different phases is needed, and moreover this needs to be achieved in three dimensions (3-D).

Using scanning electron microscopy (SEM) and energydispersive X-ray spectrometry (EDS), accurate compositions can be measured with high spatial resolution [4]. In order to obtain composition maps of the various elements, the surface of the sample is scanned. Using a focused ion beam (FIB) combined with SEM/EDS, 2-D mapping can be extended to three dimensions: using an FIB to sequentially mill away thin layers of material through the volume of interest, each section is characterized with SEM images and EDS maps. This technique is called 3-D EDS by FIB/ SEM $[5,6]$.

The precision of EDS depends on the X-ray counting statistics and is improved by long acquisition times [7]. Given the high number of spectra in a 3-D EDS stack,

\footnotetext{
${ }^{1}$ An analysis of the $\mathrm{Cr}-\mathrm{Ti}-\mathrm{Ni}$ and $\mathrm{Cr}-\mathrm{Ti}-\mathrm{Fe}$ ternary phase diagrams shows a high $\mathrm{Cr}$ solubility in $\mathrm{TiFe}_{2}$ but a low solubility in $\mathrm{Ni}_{3} \mathrm{Ti}$. Based on that, the $\mathrm{Cr}$ content is considered to be linked to that of $\mathrm{Fe}$.
}

the time spent per spectrum needs to be limited to keep the total acquisition time reasonable. Therefore the counting statistics are the main factor limiting the composition accuracy determined using this technique.

The spatial resolution of EDS is linked to the acceleration voltage, which needs to be sufficiently high to excite the desired characteristic X-ray lines. This voltage influences directly the distribution volume of the generated $\mathrm{X}$-rays that is roughly in the micrometer range [4]. Thus EDS maps have a high chemical contrast with relatively low spatial resolution. Secondary electrons (SEs) have an escape depth of less than $10 \mathrm{~nm}$. The resolution of SE images is of the same order of magnitude, considering that SEs generated at the probe location (SE1) mainly form the images [4]. With a good resolution but a low chemical contrast, SE images are complementary to EDS maps.

One aim of 3-D image processing is to segment the analyzed volume into subvolumes corresponding to chemically different phases. Two segmentation methods adapted to 3D EDS were reported: (i) a straightforward method most commonly used to isolate one phase is to set up a threshold value for one of the elements $[6,8,9]$ and (ii) a segmentation method developed by Kotula et al. [10] based on multivariate statistical analysis - this method provides a set of statistically dominant phases with their corresponding spectrum. Both of these methods rely only on EDS measurements, without using the high spatial information given by SE images.

The main goal of the present work is to characterize the complex microstructure formed in a NiTi-SS laser-welded joint, more specifically for a small region close to the NiTiweld interface where the presence of some intermetallics was revealed by back-scatter electron (BSE) contrast. 3-D EDS by FIB/SEM seemed to be the most promising characterization technique for this complex case. None of the segmentation methods found in the literature appeared appropriate; therefore a new segmentation method was developed that exploits more efficiently the complete set of data.

\section{Experimental}

\subsection{NiTi-SS welding}

The sample used for the present investigation was produced by welding wires of NiTi $(50.8$ at. $\% \mathrm{Ni}$ ) and $\mathrm{SS}$ (grade $304 \mathrm{~L}$ ), $300 \mu \mathrm{m}$ in diameter. Prior to welding, NiTi wires were chemically etched in a hydrofluoric and nitric acid aqueous solution to remove the titanium black oxide layer formed during their production, and SS wires were used as received (wire-drawn without annealing). Welding was performed using a Nd:YAG pulsed laser coupled to an orbital welder. The pulse energy and duration were adjusted to achieve a complete transverse weld. The time between each pulse was set to be long enough to allow solidification and heat diffusion over the wire radius between each pulse, and thus to obtain isotherms almost 
normal to the wire axis, right at the beginning of the next pulse. A laminar flux of pure argon was blown during welding in order to avoid titanium oxidation. More details about the welding conditions can be found in Refs. [1,11].

A longitudinal section of a welded specimen observed by $\mathrm{SEM} / \mathrm{BSE}$ is shown in Fig. 2. Prior to the observation, the section was mechanically polished with diamond lapping grinding disks and aqueous silica solution to obtain a mirror-polished section.

In Fig. 2, the SS is on the left and the NiTi is on the right. The weld made by a succession of spot welds around the periphery of the two wires appears as a "diabolo" shape in this section. Slight compositional variations within the weld are responsible for the various gray levels and clearly show that Marangoni convection during the laser spots is not sufficient to achieve complete mixing in the liquid. A thin layer (white arrows) is observed at the interface between the weld and the NiTi wire. The white rectangle shows the location analyzed by 3-D EDS.

\subsection{3-D EDS by FIB/SEM}

The 3-D data were collected with a Carl Zeiss Nvision40. This microscope comprises a vertical electron column and an ion column (Liquid Metal Ion Source, $\mathrm{Ga}^{+}$) with an angle of $54^{\circ}$ in between. The EDS detector, an Oxford Instrument X-Max with $80 \mathrm{~mm}^{2}$ detection area, is mounted at an azimuthal angle of $90^{\circ}$ and an elevation angle of $37^{\circ}$.

Prior to the 3-D run, the stage was tilted $54^{\circ}$ in order to have the ion beam normal to the sample surface, as shown in Fig. 3. A protective layer of carbon was deposited on the top of the region of interest, in order to minimize curtaining effects [12]. Trenches were milled around the deposited layer to gain access to the volume of interest. A freshly milled section was obtained perpendicular to the surface. The electron beam images the section with an angle of $36^{\circ}$ and the resulting take-off angle for the EDS detector is $27^{\circ}$ [6]. During the 3-D acquisition, layers were etched

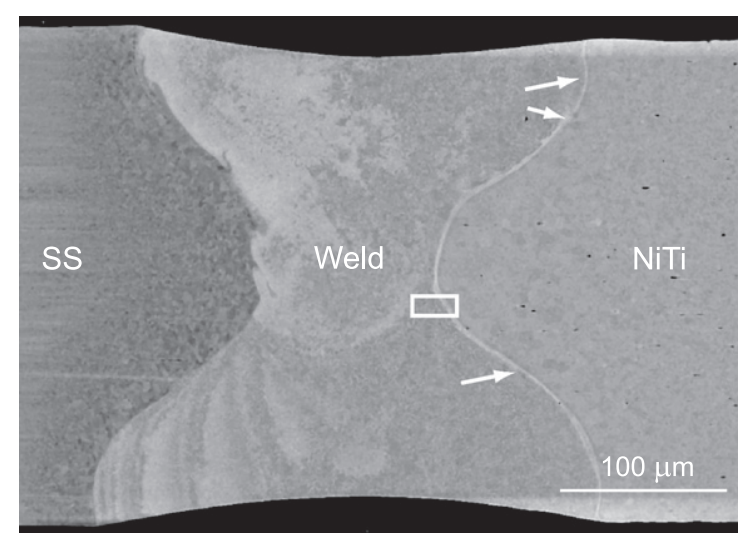

Fig. 2. SEM back-scattered image of a longitudinal cut through a welded NiTi-SS couple. The white rectangle indicates the region of 3-D EDS acquisition. Arrows show the thin intermetallic layer of interest.

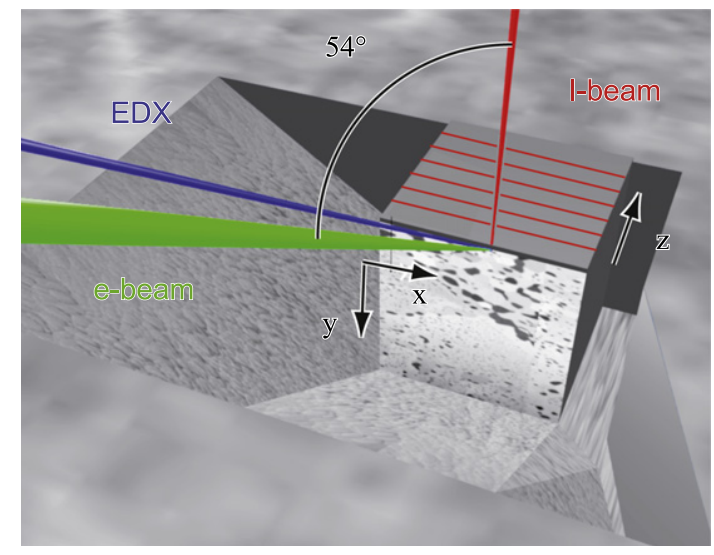

Fig. 3. Schematic view of a sample ready for acquisition. The position of the different beams is shown as well as the angle between the ion beam and the electron beam. The $x, y$ and $z$ axes are defined for this type of geometry. The red lines show the position of the subsequent sections.

away sequentially through the whole volume. A SE image of the section was recorded after each removed slice, i.e. in this case $12.5 \mathrm{~nm}$. An EDS map was acquired after every eighth slice had been removed, i.e. $100 \mathrm{~nm}$ under the present conditions.

Table 1 gives the set of the lowest-energy X-ray lines without overlaps. The acceleration voltage was set to $10 \mathrm{kV}$ in order to excite efficiently these X-ray lines.

The depth distributions of the emitted X-rays were simulated by Monte Carlo simulations. Fig. 4 shows the distributions for the three main elements (Fe, Ni, Ti). Each X-ray line is simulated in the corresponding pure material. The maximum X-ray production depth for the three elements ranges from 200 to $450 \mathrm{~nm}$. Most of the X-rays are generated in the first $100 \mathrm{~nm}$. For both EDS maps and SE images, the pixel sizes in $x$ and $y$ were set to have isometric voxels $\left(100 \times 100 \times 100 \mathrm{~nm}^{3}\right.$ and $12.5 \times 12.5 \times 12.5 \mathrm{~nm}^{3}$, respectively). Each EDS map was scanned through with $128 \times 96$ points, while SE images comprise $1024 \times 768$ points.

A period of 6 min was spent per EDS map, thus $30 \mathrm{~ms}$ per spectrum recorded at each pixel. SEM current $(2.8 \mathrm{nA})$ and EDS detector process time were optimized to obtain an average output count rate of $40 \mathrm{kcps}$ (at a dead time of $40 \%$ ). The 3-D acquisition was run through a volume of $12.8 \times 9.6 \times 4.4 \mu^{3}$ at the NiTi-weld interfacial region (white rectangle in Fig. 2). During the $12 \mathrm{~h}$ acquisition, 352 SE images and 44 EDS maps were subsequently recorded.

In order to assess the precision of the short EDS maps of the 3-D runs, a longer 2-D EDS map with better statistics

Table 1

$\mathrm{X}$-ray lines and energies for the analyzed elements.

\begin{tabular}{llllll}
\hline & $\mathrm{C}$ & $\mathrm{Ti}$ & $\mathrm{Cr}$ & $\mathrm{Fe}$ & $\mathrm{Ni}$ \\
\hline Lines & $\mathrm{K} \alpha$ & $\mathrm{K} \alpha$ & $\mathrm{K} \alpha$ & $\mathrm{K} \alpha$ & $\mathrm{L} \alpha$ \\
$E(\mathrm{keV})$ & 0.28 & 4.51 & 5.41 & 6.40 & 0.85 \\
\hline
\end{tabular}




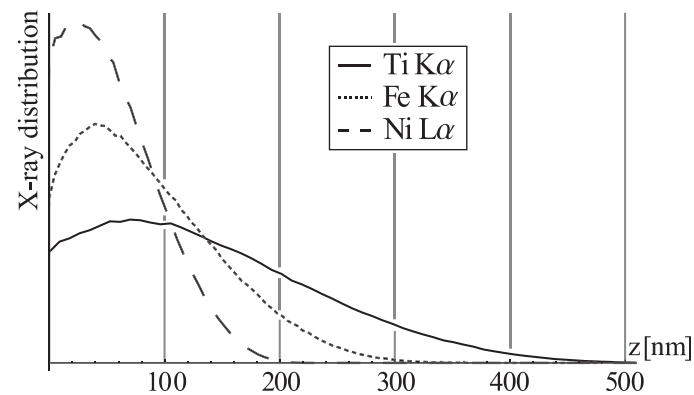

Fig. 4. Depth distributions of emitted X-rays at $10 \mathrm{kV}$ for the main three elements of NiTi-SS welds. Each distribution is simulated in the corresponding pure material: $\mathrm{Ti} \mathrm{K} \alpha$ in $\mathrm{Ti}, \mathrm{Fe} \mathrm{K} \alpha$ in $\mathrm{Fe}$, and $\mathrm{Ni} \mathrm{L} \alpha$ in $\mathrm{Ni}$.

was recorded prior to the 3-D acquisition with the same microscope parameters. The dwell time was chosen to be 10 times longer ( $300 \mathrm{~ms}$ dwell time, $1 \mathrm{~h}$ for the whole map).

The standard spectra used for quantification were recorded with the same microscope parameters, apart from a live time of $30 \mathrm{~s}$. NiTi base wire was used as a microanalysis standard for $\mathrm{Ni}$ and $\mathrm{Ti}$, as the ratio between this two elements is certified, and impurities are guaranteed below $0.1 \mathrm{wt} . \%$. For the other analyzed elements, pure materials mounted on a support were used (Structure Probe Incorporation supplies).

\subsection{Data processing}

The stack of $352 \mathrm{SE}$ images was registered (aligned) at a sub-pixel level using a pyramidal approach [13]. A firstorder 3-D median filter was applied to the obtained stack to reduce noise. SE images have an uneven background gray level induced by a shadow effect of the side walls. This background was reduced with a high-pass filter [14].

All EDS spectra of the 3-D run were quantified as described below. After background correction using a tophat filter, the extracted X-ray intensities of sample and standard were compared [4] and corrected for matrix effects $(\phi$ $(\rho z)$ extended Pouchou-Pichoir [15]). The obtained stacks of elemental maps were aligned based on the SE image stack alignment; the same first-order 3-D median filter was applied. Then the whole set of stacks (elemental maps and SE images) was used for segmentation. This specifically developed method is described in the Results section. To obtain a 3-D visualization, a polygonal surface representation was built with the segmented phases [16].

The same noise filter, background removal and quantification were applied to the 2-D data (SE image and EDS map).

\subsection{Software}

The simulation of X-ray emission (Fig. 4) was done with DTSA-II [17]. Stratagem software (SAMx) was used for matrix-effects correction. Before matrix correction, the spectra were processed using a home-made script. ImageJ software was used to register the images in the stack [18].
Further data processing was carried out using home-made scripts in Mathematica ${ }^{\circledR}$ 8.0. 3-D surface visualizations were obtained using Avizo ${ }^{\circledR}$ fire 6.0 (VSG).

\section{Results and discussion}

The presentation of the results is divided into four subsections. In the first part, the results of the 2-D EDS map with the longest acquisition time are presented. A new method to segment the 3-D stack is then described in the second subsection, before presenting a 3-D reconstruction of the phases. In the last subsection, the results are further discussed from a metallurgical viewpoint, with respect to the solidification and diffusion path.

\subsection{D EDS Analysis of NiTi weld interface}

Fig. 5 shows a SE image of a freshly milled section. As this section is flat, the contrast variation of the image is most probably linked to changes in phase composition. Based on EDS measurements, the black spots were identified as titanium carbides.

The elemental maps corresponding to Fig. 5, before noise filtering, are shown in Fig. 6. Even for this $1 \mathrm{~h}$ map, the time spent per pixel is quite low $(300 \mathrm{~ms})$. Considering only counting statistics, the absolute errors $(1 \sigma)$ range from 2 to 5 at.\%, depending mainly on the intensity of the $\mathrm{X}$-ray line, and appear as noise in the elemental maps. To obtain an accurate composition, the X-ray intensities are corrected for matrix-effects (absorption and fluorescence). The correction method used is based on a locally homogeneous composition. To correct for the absorption, the homogeneity range needs to be within the volume of the primary generated X-rays (and in the direction to the detector), in the present case around $300 \mathrm{~nm}$. For the fluorescence correction, the homogeneity range needs to be bigger, but this is a weaker correction as observed in the present case with calculations with and without fluorescence correction. Analyses close to the phase boundary have a slightly lower level of accuracy. The total volume

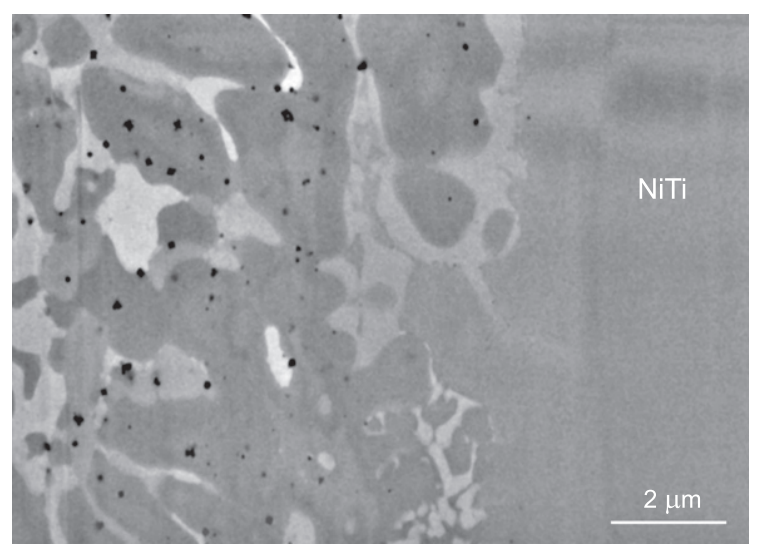

Fig. 5. SEM secondary electron image of a 2-D section of a NiTi-weld interfacial region. 

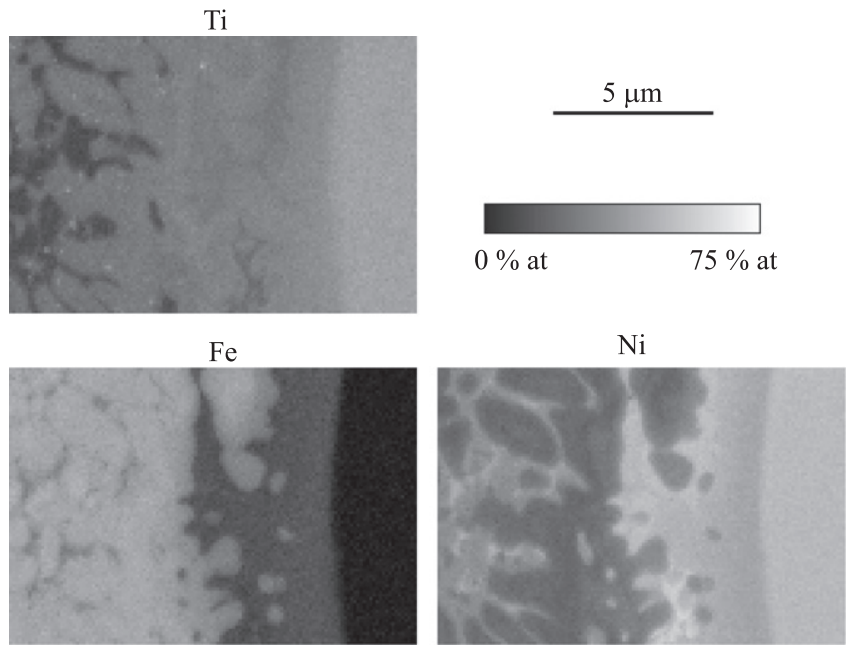

Fig. 6. Quantitative X-ray maps of Ti, Fe and Ni for the section shown in Fig. 5 and a $1 \mathrm{~h}$ acquisition time. The gray scale gives the concentration in at.\%. The NiTi wire is on the right.

of these regions is small, so that from a statistical point of view the composition of the different phases remains accurate.

The Fe, $\mathrm{Ni}$ and $\mathrm{Ti}$ compositions measured at each pixel of the 2-D section are reported in the ternary Gibbs simplex in Fig. 7, on a logarithmic scale (from blue to red) [19]. This composition histogram is overlaid on a projection of the liquidus in (a), while in (b) the same histogram is shown with a $1000^{\circ} \mathrm{C}$ isothermal section of the $\mathrm{Fe}-\mathrm{Ni}-\mathrm{Ti}$ phase diagram [2]. The composition "path" can be described as follows: (i) from NiTi on the right of the Gibbs simplex, the compositions then follow the bottom limit of the $(\mathrm{Fe}, \mathrm{Ni}) \mathrm{Ti}$ region; (ii) once the end of the $(\mathrm{Fe}, \mathrm{Ni}) \mathrm{Ti}$ tie-line is reached in Fig. 7, the path goes toward the $\mathrm{Ni}_{3} \mathrm{Ti}$ intermetallics; (iii) the path then follows the tie-line between $\mathrm{Ni}_{3} \mathrm{Ti}$ and $\mathrm{Fe}_{2} \mathrm{Ti}$; (iv) finally, the path crosses the ternary region toward $\gamma-(\mathrm{Fe}, \mathrm{Ni})$. These results and what they imply in terms of the solidification and diffusion paths are further discussed in the following section based on 3-D stack segmentation.

\subsection{3-D stacks segmentation}

As for the 2-D case, ternary composition histograms were calculated from the 3-D stacks of elemental maps, cutting the lowest and highest frequency counts at 100 cts and $25 \mathrm{kcts}$, respectively. Compared to Fig. 7, the peaks and the composition path deduced from the 3-D stack are significantly broader in Fig. 8. This is due to the lower number of counts (10 times fewer counts). Nevertheless, the global shape of the diffusion path is preserved and different intermetallic regions are distinct enough to be used as a starting point for the segmentation.

In a first approach, threshold values on composition were defined for each of the three main elemental maps according to:

$t_{\min , i} \leqslant C_{i}<t_{\max , i} \quad$ where $i=\mathrm{Fe}, \mathrm{Ni}, \mathrm{Ti}$

where $t_{\min , i}$ and $t_{\max , i}$ correspond to the minimum and maximum threshold values, respectively, of the corresponding composition $C_{i}$. On the ternary diagram of Fig. 8, these threshold values form lines that define the edges of a threshold polygonal domain. The latter can have a maximum of six edges parallel to the boundaries of the Gibbs simplex. If no constraint is given for a particular element composition (i.e. $t_{\text {min }, i}=0$ and $t_{\max , i}=\infty$ ), the threshold domain becomes a parallelogram with $60^{\circ}$ and $120^{\circ}$ angles. An

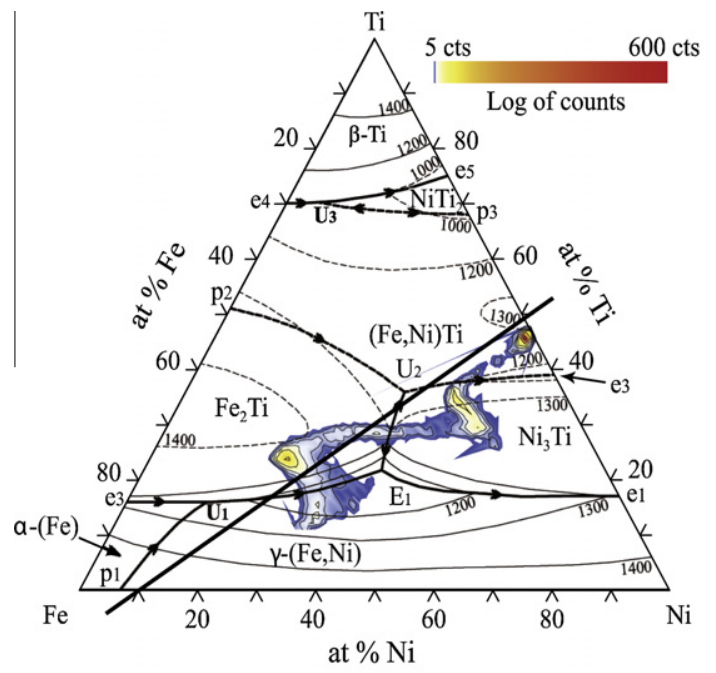

(a)

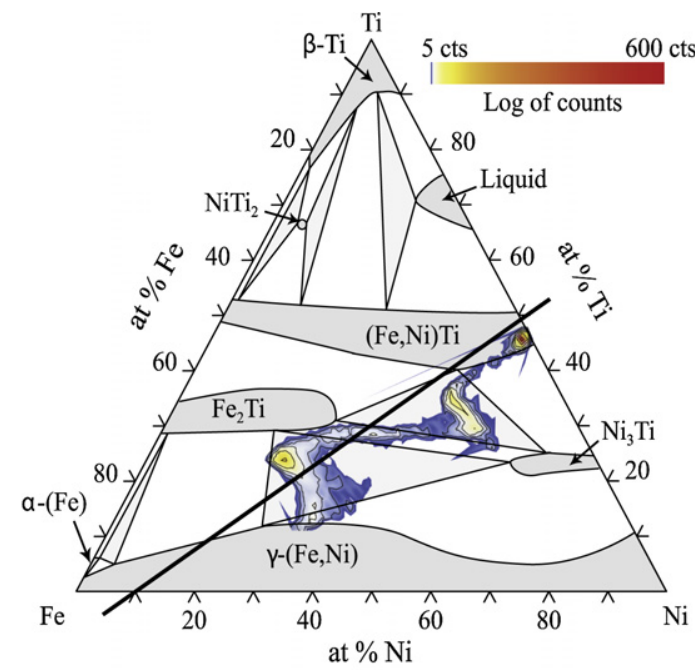

(b)

Fig. 7. Histogram of the compositions measured by 2-D EDS in the section shown in Fig. 5 and displayed in the Fe-Ni-Ti Gibbs simplex. This histogram is superimposed on a projection of the liquidus in (a) and on a $1000^{\circ} \mathrm{C}$ isothermal section of the Fe-Ni-Ti phase diagram in (b) [2]. The logarithm color code used to scale the three compositions is shown in the top right corner. 


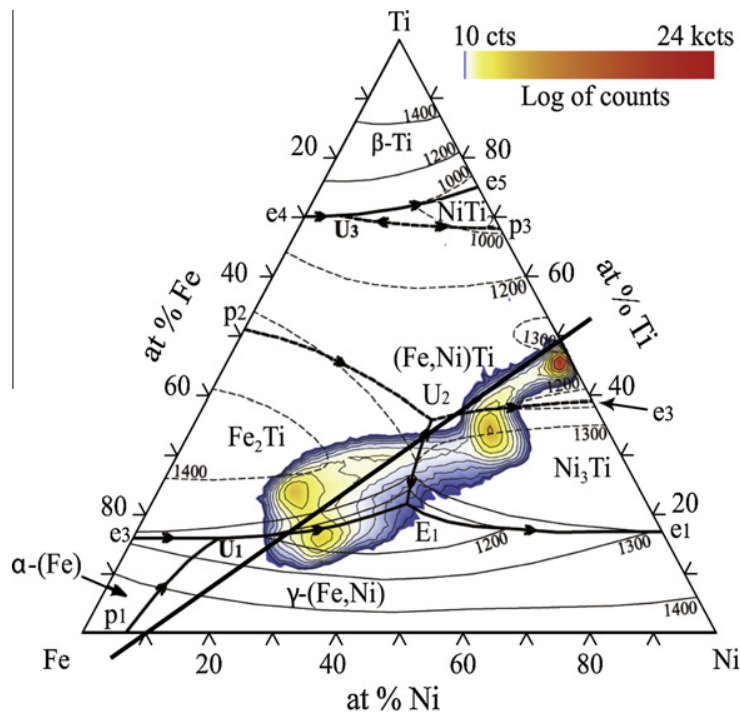

(a)

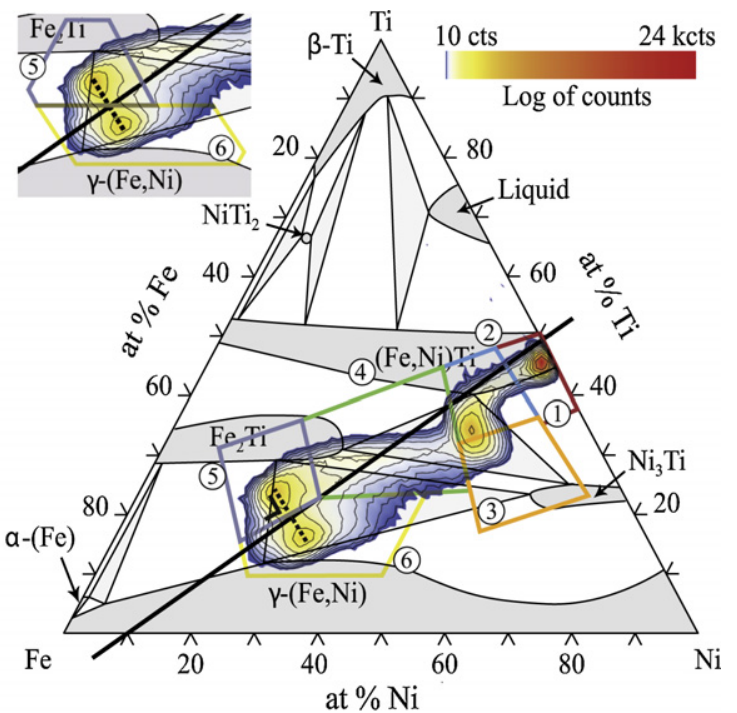

(b)

Fig. 8. Ternary histogram of the Ti, Ni and Fe compositions from 3-D stack of 44 EDS maps (6 min each), superimposed on the projection of the liquidus in (a) and the $1000^{\circ} \mathrm{C}$ isothermal section of the $\mathrm{Fe}-\mathrm{Ni}-\mathrm{Ti}$ phase diagram in (b) [2]. The logarithm scale for this histogram is shown at the top right corner. In (b), the labeled polygons give the threshold used for segmentation, and the inset shows a part of the ternary histogram with threshold values set parallel to the three main axes of the Gibbs simplex.

example of two threshold domains defined by Eq. (1) is shown in the inset of Fig. 8b for regions corresponding primarily to $\mathrm{Fe}_{2} \mathrm{Ti}$ (region 5) and $\gamma-(\mathrm{Fe}, \mathrm{Ni}$ ) (region 6). The dashed line drawn between these two regions links the two maxima of the composition histogram and goes through a saddle point in between. The ternary histogram shows a symmetry with respect to this line, but the boundary separating these two domains does not respect this symmetry since it is parallel to the edges of the Gibbs simplex and not perpendicular to this line. This will lead to an incorrect segmentation for regions of the 3-D stack having compositions close to the boundary between regions 5 and 6 .

In order to be able to define domain boundaries, which are not necessarily parallel to the edges of the Gibbs simplex, we can define lines that satisfy a constraint of the type $\left(A_{F e} C_{F e}+A_{N i} C_{N i}+A_{T i} C_{T i}\right)=B$, where the $A$ and $B$ are parameters, in addition to the condition $\left(C_{F e}+C_{N i}+\right.$ $\left.C_{T i}\right)=1$. Alternatively, one can define for each composition $C_{i}$ two parameters, $d_{\min , i}$ and $d_{\max , i}$, such that:

$$
\begin{aligned}
& t_{\text {min }, i} \leqslant C_{i}-d_{\text {min }, i} C_{j} \\
& t_{\text {max }, i}>C_{i}-d_{\text {max }, i} C_{j} \text { where } i, j=\mathrm{Fe}, \mathrm{Ni}, \mathrm{Ti}
\end{aligned}
$$

with $i$ different from $j$. The factors $d_{\min , i}$ and $d_{\max , i}$ thus allow rotation of the boundaries of the threshold domain so that they are perpendicular to the "path" of the composition histogram as shown in the overall Gibbs simplex of Fig. 8b.

According to the measured compositions and the phase diagram, four phases are involved in this region of the solidified weld: the NiTi wire on the right of the Gibbs simplex and its associated solid solution $(\mathrm{Fe}, \mathrm{Ni}) \mathrm{Ti}, \mathrm{Ni}_{3} \mathrm{Ti}$, $\mathrm{Fe}_{2} \mathrm{Ti}$, and $\gamma-(\mathrm{Fe}, \mathrm{Ni})$. These four phases can appear as single-phase composition domains in the microstructure if they can be resolved with the present segmentation technique, or as a mixture of two or more phases if such is not the case (e.g. a fine eutectic or eutectoid region). In the present case, six composition domains identified in Fig. 8b (domains 1-6) have been segmented with this approach. Their composition domain boundaries coincide with those of neighboring domains in order to have all the voxels attributed. These domains are now discussed in terms of solidification and diffusion paths.

The NiTi base wire, which was not melted during welding, appears on the right of the SE image contrast of Fig. 5: it is identified as composition domain 1 in Fig. 8b. Next to it (domain 2), one finds a solid solution (Fe, Ni) Ti identical in crystallographic structure to NiTi but enriched in Fe. Considering the liquidus projection of $(\mathrm{Fe}, \mathrm{Ni}) \mathrm{Ti}$ (Fig. 8a), this surface exhibits a maximum in temperature near the center of the Gibbs simplex. Since the line joining the NiTi wire to $\gamma-(\mathrm{Fe}, \mathrm{Ni})$ does not cross this maximum, the solidification path remains on the side of the peritectic point U2 and does not go towards the other side U3. The $(\mathrm{Fe}, \mathrm{Ni}) \mathrm{Ti}$ phase starts its growth from the unmelted NiTi wire on the side of the melt pool as a planar front (since the velocity is zero at the weld pool trace), then rapidly develops cells and dendrites as the velocity increases. Once the eutectic monovariant line $\mathrm{U} 2-\mathrm{e} 3$ is reached, the $\mathrm{Ni}_{3} \mathrm{Ti}$ phase can form (domain 3). Note that the maximum of composition in Fig. 8a is very close to the monovariant line U2-e3 and thus probably corresponds to an unresolved eutectic morphology comprised of $(\mathrm{Fe}, \mathrm{Ni}) \mathrm{Ti} / \mathrm{Ni}_{3} \mathrm{Ti}$ lamellae or fibers.

Considering now the Fe-rich side of the line joining NiTi to $\gamma$-iron, it can be seen from Fig. 8a that the $\mathrm{Fe}_{2} \mathrm{Ti}$ phase can form at higher temperature (between 1300 and 
$1400{ }^{\circ} \mathrm{C}$ ). It can then form in the Fe-rich melt pool, ahead of the $(\mathrm{Fe}, \mathrm{Ni}) \mathrm{Ti} / \mathrm{Ni}_{3} \mathrm{Ti}$ eutectic. This corresponds to domain 5 in Fig. 8b. As it grows, the composition path reaches the eutectic monovariant line $\mathrm{U} 1-\mathrm{E} 1$ and $\gamma-(\mathrm{Fe}, \mathrm{Ni})$ can form, corresponding to domain 6 . Again, the maximum of this composition domain is close to the monovariant line and thus this domain is probably a eutectic comprised of both $\gamma-(\mathrm{Fe}, \mathrm{Ni})$ and $\mathrm{Fe}_{2} \mathrm{Ti}$. Finally, the microstructure formed from the NiTi wire (domains 2 and 3 ) and the one nucleating and growing in the melt (domains 5 and 6) meet at a region close to the monovariant line U2-E1. Note that this line has a saddle point and that the corresponding composition domain 4 seems biased toward the ternary eutectic point E1.

The limits of the threshold domains (factors $t$ in Eq. (2)) were finely tuned so as to approach the gray level boundary of the SE images. Orthogonal views through the middle of the stack are shown in Fig. 9. SE gray level images are overlaid with the six colored composition domains (1-4-5

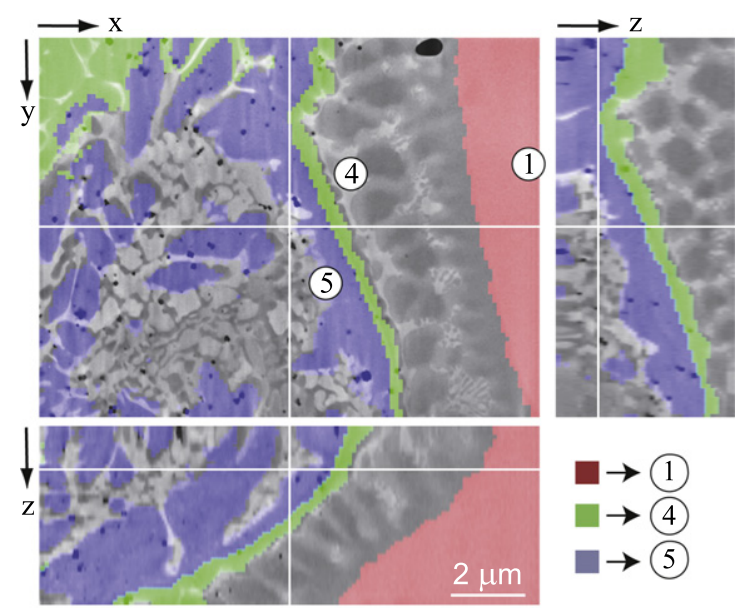

(a)

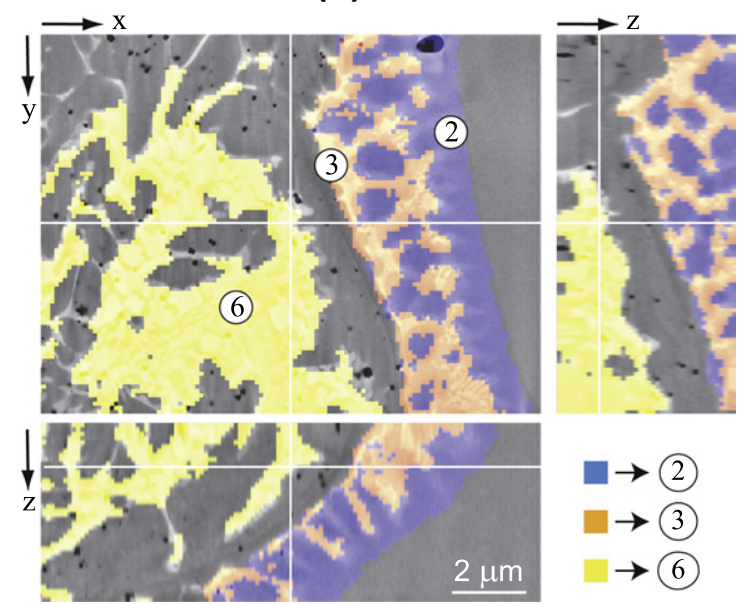

(b)

Fig. 9. Orthogonal views of SE images with superimposition of the colored composition domains $1-6(1-4-5$ in (a), 2-3-6 in (b)). Thresholds for the six composition domains are defined in Fig. 8. The white lines show the position of the other orthogonal views. at the top, 2-3-6 at the bottom). Complex fine structures such as the interface between domains 2 and 3 or the two contrasts in the SE image in domain 6 cannot be resolved with EDS maps.

\subsection{Segmentation refinement}

A second segmentation step is applied considering SE images in order to go beyond the spatial resolution limit of EDS maps. Two regions with a fine microstructure were treated in this way, the composition domain 6 , which is suspected of being a $\gamma-(\mathrm{Fe}, \mathrm{Ni}) / \mathrm{Fe}_{2} \mathrm{Ti}$ eutectic, and domains 2 and 3, which most likely correspond to $(\mathrm{Fe}, \mathrm{Ni}) \mathrm{Ti}$ dendrites and to a $(\mathrm{Fe}, \mathrm{Ni}) \mathrm{Ti} / \mathrm{Ni}_{3} \mathrm{Ti}$ eutectic, respectively. These regions were isolated from the rest of the SE images using the composition domains of Fig. 9 as masks. The gray level of the SE images allows one to finely segment these regions into subdomains which might correspond to individual phases of each eutectic (see Fig. 10). A very good example of the higher resolution that can be achieved is provided by the bottom region of domain 3 in the $x--y$ orthogonal slice of Fig. 9: it is a nearly uniform orange zone in this figure, whereas in Fig. 10, it clearly appears as a eutectic zone made of blue (zone $2,(\mathrm{Fe}, \mathrm{Ni}) \mathrm{Ti}$ ) and orange (zone $3, \mathrm{Ni}_{3} \mathrm{Ti}$ ). Similarly, domain 6 , which was a uniform yellow region in Fig. 9, is now a yellow and red composite in Fig. 10, corresponding to $\gamma-(\mathrm{Fe}, \mathrm{Ni})$ and $\mathrm{Fe}_{2-}$ $\mathrm{Ti}$, respectively. This correspondence is confirmed by EDS measurements in the yellow region (subdomain 6a) that shows a lower $\mathrm{Ti}$ content than the ones in the red region (subdomain 6b).

Fig. 11 shows a flowchart that summarizes the segmentation method. First, a ternary histogram is generated from the elemental maps. In order to isolate chemically similar regions, threshold domains are defined on this histogram. The limits of these domains are then finely tuned with the help of phase visualization overlaying SE images. Further

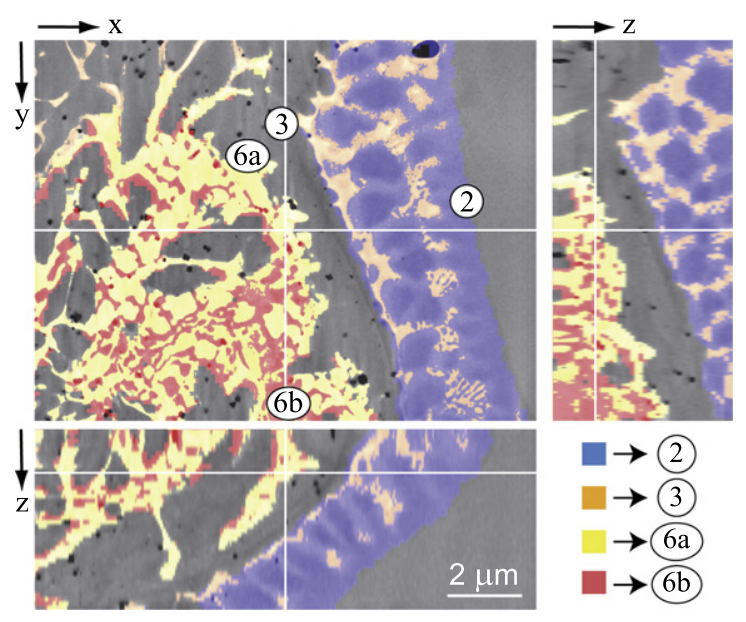

Fig. 10. Orthogonal views of SE images with refined domains (2, 3, 6a and 6b) superimposed. The domains of Fig. 9 are refined with SE image contrast. The white lines show the position of the other orthogonal views. 


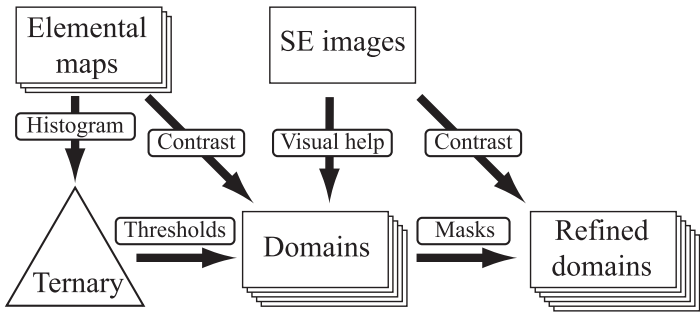

Fig. 11. Flowchart of the segmentation method. First row: input stacks. Second row: segmentation steps. Arrows: processes.

refinement into subdomains is obtained by applying thresholds on the SE images.

\subsection{Phase formation}

The seven regions, 1-6 with a further distinction of $6 \mathrm{a}$ and $6 \mathrm{~b}$, obtained with the refined segmentation (Figs. 9 and 10) can be used for 3-D visualization. A surface reconstruction of the seven domains is presented in Fig. 12. In this section, the phase formation is summarized based on this visualization.

This 3-D reconstruction of the phase formation sequence made by FIB slicing and EDS mapping analysis illustrates well the solidification and diffusion paths followed by the weld pool during solidification. At the end of the laser pulse, when solidification starts, the weld is completely molten and only the NiTi base wire (phase 1) is solid in the analyzed volume. The first phase to form during solidification is the (Fe, Ni) Ti phase (phase 2), which grows epitaxially from the NiTi wire, first with a planar front morphology, then as cells/dendrites. Once the U2e3 eutectic line is reached, the remaining liquid in between the dendrite arms solidifies as a eutectic with the formation of the second-phase $\mathrm{Ni}_{3} \mathrm{Ti}$ (region 3). In the Fe-rich part of the weld, the $\mathrm{Fe}_{2} \mathrm{Ti}$ phase forms at higher temperature by nucleation and growth from the melt (domain 5). In between the dendrite arms, the Ti-poor melt solidifies as a $\gamma$-(Fe, $\mathrm{Ni}) / \mathrm{Fe}_{2} \mathrm{Ti}$ eutectic (regions $6 \mathrm{a}$ and $6 \mathrm{~b}$ ), following the monovariant line U1-E1. Finally, when regions 5-6 and 2-3 meet, region 4 forms, probably as a ternary eutectic E1.

\section{Conclusion}

In this research, the complex microstructure formed in a NiTi-SS laser-welded joint was studied with 3-D EDS by FIB/SEM. With this technique, a 3-D stack of elemental distribution maps (from EDS) and SE images was acquired. To identify all the phases with sufficiently high accuracy, a new segmentation method that uses both composition maps and SE images of higher spatial resolution was developed.

A first segmentation was done based on the measured compositions and on the ternary $\mathrm{Fe}-\mathrm{Ni}-\mathrm{Ti}$ phase diagram. Individual phases corresponding to fairly coarse structures such as the base NiTi wire or dendrites could be directly identified. In other cases such as eutectics, the microstructure was too fine to be resolved with EDS maps only. A refined segmentation based on SE images was then undertaken to isolate individual phases in otherwise unresolved EDS elemental maps. Phases appearing in two eutectics were clearly identified this way.

Using a 3-D visualization of the segmented phases, a careful analysis of the NiTi-weld interface revealed the absence of an intrinsic layer at the crack nucleation site. The appearance sequence of the various phases/morphologies revealed by the present analysis could be clearly correlated with the ternary $\mathrm{Fe}-\mathrm{Ni}-\mathrm{Ti}$ phase diagram and with the solidification phenomena occurring during single-pulse laser welding.

\section{Acknowledgments}

The microscopy part of this research was sponsored by Carl Zeiss, the material part by the Swiss Confederations's Innovation Promotion Agency CTI (Project No. 8545.1).

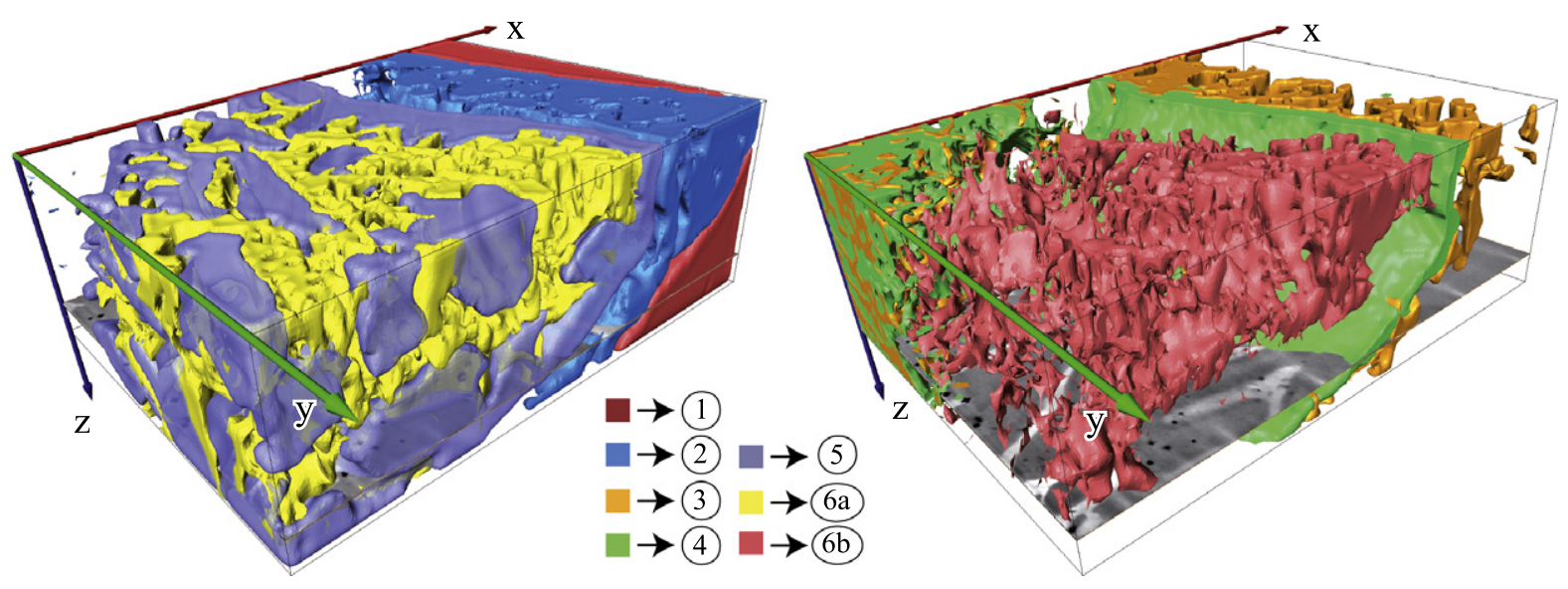

Fig. 12. Surface reconstruction of the seven regions identified by combined FIB, EDS and SE. Region 1: unmelted NiTi wire; region 2: (Fe, Ni) Ti dendrites; region $3: \mathrm{Ni}_{3} \mathrm{Ti}$ formed in the U2-e3 eutectic; region 4: thin region (probably a mixture of phases corresponding to the ternary eutectic E1); region 5: $\mathrm{Fe}_{2} \mathrm{Ti}$ dendrites; region 6: eutectic made of $\gamma$-(Fe, $\mathrm{Ni}$ ) in yellow (a) and $\mathrm{Fe}_{2} \mathrm{Ti}$ in red (b). 
Oxford Instruments is thanked for its technical support. Heraeus Medical Components Division is thanked for providing the wires and laser facility.

\section{References}

[1] Vannod J, Bornert M, Bidaux J, Bataillard L, Karimi A, Drezet J, et al. Acta Mater 2011;59:6538.

[2] Cacciamani G, De Keyzer J, Ferro R, Klotz U, Lacaze J, Wollants P. Intermetallics 2006;14:1312.

[3] Kirkaldy JS, Brown LC. Can Metall Quart 1963;2:89.

[4] Goldstein J, Newbury DE, Echlin P, Joy DC, Lyman CE, Lifshin E, et al. Scanning electron microscopy and X-ray microanalysis. Springer; 2003.

[5] Kotula P, Keenan M, Michael J. Microsc Microanal 2003;9:1.

[6] Schaffer M, Wagner J, Schaffer B, Schmied M, Mulders H. Ultramicroscopy 2007;107:587.

[7] Lifshin E, Doganaksoy N, Sirois J, Gauvin R. Microsc Microanal 1998;4:598.
[8] Lasagni F, Lasagni A, Marks E, Holzapfel C, Mncklich F, Degischer H. Acta Mater 2007;55:3875.

[9] Scott K. J Microsc 2011;242:86.

[10] Kotula PG, Sorensen NR. JOM 2011;63:41.

[11] Vannod J. Laser welding of nickel-titanium and stainless steel wires: processing, metallurgy and properties. Ph.D. thesis, Ecole Polytechnique FTdTrale de Lausanne, Switzerland; 2011.

[12] Giannuzzi LA, Stevie FA. Introduction to focused ion beams. Springer; 2005.

[13] ThTvenaz P, Ruttimann U, Unser M. IEEE Trans Image Process 1998;7:27.

[14] Russ JC. The image processing handbook. CRC Press; 2007.

[15] Pouchou JL, Pichoir F. In: Heinrich KFJ, Newbury DE, editors. Electron probe quantitation. Plenum Press; 1992.

[16] Ohser J, Schladitz K. 3D images of materials structures: processing and analysis. Wiley $\mathrm{VCH} ; 2009$.

[17] Ritchie N. Surf Interface Anal 2005;37:1006.

[18] Abramoff MD. Biophotonics Int 2004;11:42.

[19] Bright D, Newbury D. Anal Chem 1991;63:243A. 